\title{
Histamine-2 receptor antagonists versus proton pump inhibitors for septic shock after lower gastrointestinal tract perforation: a retrospective cohort study using a national inpatient database
}

\author{
Jun Suzuki ${ }^{1 *}$ D, Yusuke Sasabuchi ${ }^{2}$, Shuji Hatakeyama ${ }^{1,3}$, Hiroki Matsui ${ }^{4}$, Teppei Sasahara ${ }^{1,5}$, Yuji Morisawa ${ }^{1}$,
} Toshiyuki Yamada ${ }^{6}$ and Hideo Yasunaga ${ }^{4}$

\begin{abstract}
Background: Studies have shown the potential benefit of stress ulcer prophylaxis including histamine-2 receptor antagonists (H2RA) and proton pump inhibitors (PPI) in critically ill patients. However, the adverse effects of stress ulcer prophylaxis such as Clostridioides difficile infection (CDI) and hospital-acquired pneumonia have been reported. Abdominal septic shock is associated with increased risk of bleeding, CDI, and pneumonia; however, which ulcer prophylaxis might be associated with better outcomes in patients with septic shock after lower gastrointestinal tract perforation is unknown.

Methods: In this retrospective cohort study using the Japanese Diagnosis Procedure Combination database from July 2010 to March 2015, we identified patients aged 18 years or older who received open abdominal surgery for lower gastrointestinal tract perforation and who used vasopressors and antibiotics within 2 days of admission. We performed propensity score matching and inverse probability of treatment weighting (IPTW) to compare the outcomes between patients who received H2RA and those who received PPI within 2 days of admission. The outcomes included gastrointestinal bleeding requiring endoscopic hemostasis within 28 days of admission, 28-day mortality, CDI, and hospital-acquired pneumonia.

Results: The propensity score matching created 1088 pairs of patients who received H2RA or PPI within 2 days of admission. There were no significant differences between the H2RA and PPI groups regarding gastrointestinal bleeding requiring endoscopic hemostasis within 28 days of admission $(0.74 \%$ vs $1.3 \%$, risk ratio $0.57(0.24-1.4)$, and $P=0.284)$, 28 -day mortality $(11.3 \%$ vs $12.9 \%$, risk ratio $0.88(0.68-1.1)$, and $P=0.386)$, CDI $(0.64 \%$ vs $0.46 \%$, risk ratio $1.4(0.45-4.4)$, and $P=0.774)$, and hospital-acquired pneumonia (3.0\% vs $4.3 \%$, risk ratio $0.70(0.45-1.1)$, and $P=$ 0.138). IPTW analysis showed similar results.

(Continued on next page)
\end{abstract}

\footnotetext{
* Correspondence: j-suzuki@jichi.ac.jp

'Division of Infectious Diseases, Jichi Medical University Hospital, 3311-1

Yakushiji, Shimotsuke, Tochigi 329-0498, Japan

Full list of author information is available at the end of the article
}

(c) The Author(s). 2020 Open Access This article is licensed under a Creative Commons Attribution 4.0 International License, which permits use, sharing, adaptation, distribution and reproduction in any medium or format, as long as you give appropriate credit to the original author(s) and the source, provide a link to the Creative Commons licence, and indicate if changes were made. The images or other third party material in this article are included in the article's Creative Commons licence, unless indicated otherwise in a credit line to the material. If material is not included in the article's Creative Commons licence and your intended use is not permitted by statutory regulation or exceeds the permitted use, you will need to obtain permission directly from the copyright holder. To view a copy of this licence, visit http://creativecommons.org/licenses/by/4.0/. The Creative Commons Public Domain Dedication waiver (http://creativecommons.org/publicdomain/zero/1.0/) applies to the data made available in this article, unless otherwise stated in a credit line to the data. 
(Continued from previous page)

Conclusions: There were no significant differences in gastrointestinal bleeding requiring endoscopic hemostasis within 28 days of admission, 28-day mortality, CDI, and hospital-acquired pneumonia between H2RA and PPI in patients with septic shock after lower gastrointestinal tract perforation.

Keywords: Bleeding, Clostridioides difficile infection, Histamine-2 receptor antagonists, Mortality, Hospital-acquired pneumonia, Proton pump inhibitors, Peritonitis

\section{Background}

Septic shock after lower gastrointestinal tract perforation is one of major causes of abdominal infection, and the mortality has been reported to be 18 to $50 \%$ [1-3]. Clostridioides difficile (formerly Clostridium difficile) infection (CDI) and pneumonia are common complications after abdominal infection [4-6], and these complications are associated with higher mortality in patients with septic shock after lower gastrointestinal tract perforation [7, 8].

Histamine-2 receptor antagonists (H2RA) and proton pump inhibiter (PPI) play an essential role in stress ulcer prophylaxis in patients with septic shock $[9,10]$. There are several concerns that stress ulcer prophylaxis was associated with CDI [11] and hospital-acquired pneumonia [12]. Previous systematic review and meta-analysis suggested that PPI and H2RA showed similar risk of hospital-acquired pneumonia or CDI [13, 14]. However, clinical heterogeneity between studies included in these meta-analyses was substantial. On the other hand, a retrospective cohort study suggested that the frequency of adverse effects of H2RA may differ from those of PPI [14]. If the risk of CDI or hospital-acquired pneumonia is different, a lower-risk drug should be selected. However, it is not known whether PPI and H2RA show similar risk of hospital-acquired pneumonia and CDI for patients with septic shock due to lower gastrointestinal tract perforation.

Therefore, the purpose of this study was to compare H2RA and PPI with regard to gastrointestinal bleeding, 28-day mortality, CDI, and hospital-acquired pneumonia for patients with septic shock after lower gastrointestinal tract perforation using a Japanese national inpatient database.

\section{Methods}

\section{Data source}

Data for this study were extracted from the Japanese Diagnosis Procedure Combination database, which is a nationwide administrative claims database with discharge abstracts representing approximately $50 \%$ of all admissions to acute care hospitals in Japan. The database includes the following data: (1) patient demographic data; (2) primary diagnosis, comorbidities at admission, postadmission complications during hospitalization coded with the International Classification of Diseases, 10th revision (ICD-10) and text written in Japanese language; (3) hospital identification number; (4) dates of surgery, procedures, and drug prescription; (5) discharge status (dead or alive); and (6) dates of hospital admission and discharge [15-17].

\section{Patient data}

We identified patients with septic shock after lower gastrointestinal tract perforation hospitalized between July 2010 and March 2015. We included patients who (1) were diagnosed with septic shock, (2) diagnosed with lower gastrointestinal tract perforation, (3) required open abdominal surgery within 2 days of admission, and (4) used antibiotics within 2 days of admission. Sepsis was defined as having any bacterial or fungal infection at admission based on the Angus criteria [1] (Additional Table 1). Definition of sepsis based on the Angus criteria has been validated in several DPC hospitals in Japan (the sensitivity value of 40.4, the specificity value of 83.0, and the positive predictive value of $79.8 \%$ for sepsis) [18]. Septic shock was defined as combination of diagnosis of sepsis and requirement of vasopressors within 2 days of admission [19]. Lower gastrointestinal tract perforation was identified with ICD-10 codes (K65.0, K63.1, K57.02, K57.03, K57.22, K57.23, K57.42, K57.43, K57.82, and $\mathrm{K} 57.83$ ) in the primary diagnosis or comorbidities at admission. We included patients who underwent open abdominal surgery within 2 days of admission because patients sometimes receive surgery on the next day if a patient is admitted late at night. Exclusion criteria were as follows [12]: (1) age < 18 years, (2) discharge within 2 days of admission, (3) pregnancy, (4) human immunodeficiency virus infection or acquired immunodeficiency syndrome, (5) sucralfate use within 2 days of admission, (6) medical history of peptic ulcer, (7) anticoagulant or antiplatelet drug use within 2 days of admission, (8) neither H2RA nor PPI used within 2 days of admission, and (9) both H2RA and PPI used within 2 days of admission.

\section{Study variables}

The H2RA group was defined as patients who received H2RA within 2 days of admission, whereas the PPI group was defined as patients who received PPI within 2 days of admission. Other variables included 
age, sex, ICU admission within 2 days of admission, high care unit (HCU) admission within 2 days of admission, hospital type (academic or not), hospital volume, and Japan coma scale (JCS). Age was categorized at 10 -year intervals. Hospital volume was defined as the annual mean number of patients with lower gastrointestinal tract perforation requiring open abdominal surgery. The JCS score was recorded in all patients to assess the level of consciousness on admission, and it correlated well with the Glasgow Coma Scale [20]. JCS scores were divided into 4 categories: 0 (alert), 1-3 (delirium), 10-30 (somnolence), and 100-300 (coma) $[20,21]$. The use of the following procedures within 2 days of admission were also evaluated: mechanical ventilation, intermittent and continuous renal replacement therapy, polymyxin B hemoperfusion, central venous catheter insertion, vasopressor use, transfusions (red cells, platelet concentrates, fresh-frozen plasma), antithrombin, recombinant human soluble thrombomodulin, immunoglobulin, danaparoid, hydrocortisone, primary use of antibiotics (penicillin, ampicillin, ampicillin/sulbactam, piperacillin/tazobactam, first-generation cephalosporin, second-generation cephalosporin, third-generation cephalosporin with or without effects for Pseudomonas aeruginosa, fourth-generation cephalosporin, carbapenem, aminoglycoside, fluoroquinolone, clindamycin, macrolide, tetracycline, anti-methicillin-resistant Staphylococcus aureus drugs and antifungal drugs), and two or more classes of initial antibiotic combinations.

\section{Outcomes}

Outcomes of interest in this study were gastrointestinal bleeding (ICD-10 code: K25, K26, K27, K28, K29, K920, and K922) requiring endoscopic hemostasis within 28 days of admission, 28-day mortality, CDI (ICD-10 code: A047) coded as a complication during hospitalization, and hospital-acquired pneumonia.

\section{Statistical analysis}

Descriptive statistics were presented before and after propensity score matching. Continuous variables were presented as the mean with standard deviation (SD). Categorical variables are presented as numbers with percentages.

One-to-one propensity score matching was used to adjust for differences in baseline characteristics and the severity of condition at admission between the H2RA and PPI groups. The probability that a patient received H2RA was modeled for confounders for the following characteristics: age, age category, sex, hospital type, hospital volume, comorbidities at admission, use of mechanical ventilation, intermittent and continuous renal replacement therapy, polymyxin B hemoperfusion, central venous catheter insertion, vasopressor use, transfusion, antithrombin, recombinant human soluble thrombomodulin, immunoglobulin, low molecular heparin, danaparoid, hydrocortisone, primary use of antibiotics, and use of two or more initial antibiotics. Differences between the H2RA and PPI groups before and after propensity score

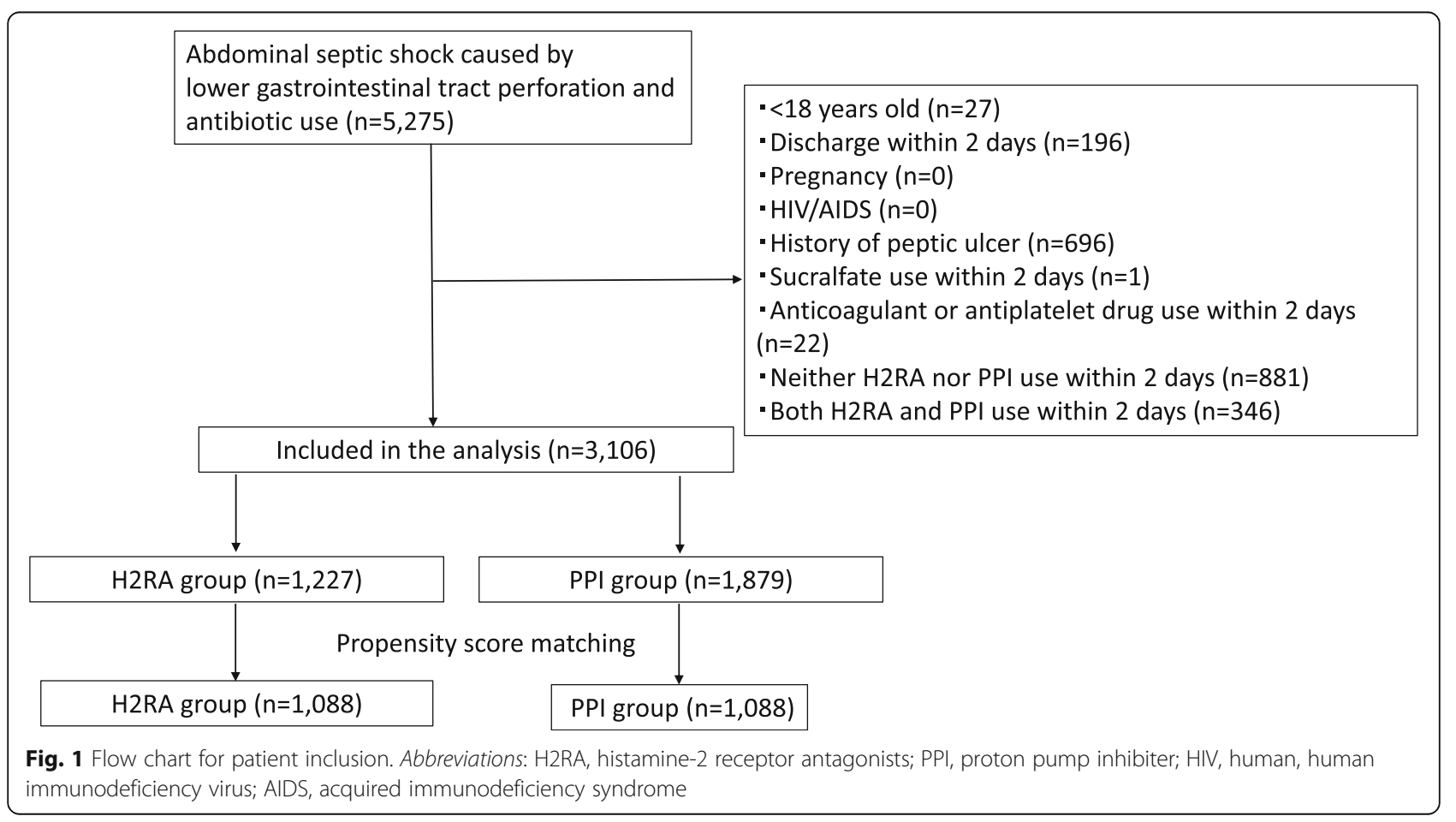


Table 1 Baseline patient characteristics in unmatched and propensity-matched groups

\begin{tabular}{|c|c|c|c|c|c|c|}
\hline \multirow[t]{3}{*}{ Variables } & \multicolumn{3}{|c|}{ Unmatched group } & \multicolumn{3}{|c|}{ Matched group } \\
\hline & \multirow{2}{*}{$\begin{array}{l}\text { H2RA group } \\
n=1227\end{array}$} & \multirow{2}{*}{$\begin{array}{l}\text { PPI group } \\
n=1879\end{array}$} & \multirow[t]{2}{*}{ SMD } & \multirow{2}{*}{$\begin{array}{l}\text { H2RA group } \\
n=1088\end{array}$} & \multirow{2}{*}{$\begin{array}{l}\text { PPI group } \\
n=1088\end{array}$} & \multirow[t]{2}{*}{ SMD } \\
\hline & & & & & & \\
\hline Age, mean (SD) & $74.5(13.1)$ & $73.7(13.0)$ & 0.06 & $74.2(13.2)$ & $74.4(12.9)$ & 0.02 \\
\hline Age category & & & 0.09 & & & 0.06 \\
\hline $10-19$ & $1(0.1)$ & $0(0.0)$ & & $1(0.1)$ & $0(0.0)$ & \\
\hline $20-29$ & $7(0.7)$ & $6(0.5)$ & & $5(0.5)$ & $4(0.4)$ & \\
\hline $30-39$ & $19(1.8)$ & $23(2.0)$ & & $19(1.7)$ & $19(1.7)$ & \\
\hline $40-49$ & $30(2.8)$ & $39(3.4)$ & & $31(2.8)$ & $27(2.5)$ & \\
\hline $50-59$ & $91(8.6)$ & $96(8.5)$ & & $82(7.5)$ & $88(8.1)$ & \\
\hline $60-69$ & $198(18.7)$ & $207(18.3)$ & & $196(18.0)$ & $196(18.0)$ & \\
\hline $70-79$ & $324(30.6)$ & $342(30.2)$ & & $308(28.3)$ & $301(27.7)$ & \\
\hline $80-89$ & $326(30.8)$ & $365(32.3)$ & & $364(33.5)$ & 366 (33.6) & \\
\hline 90-99 & $62(5.9)$ & $52(4.6)$ & & $80(7.4)$ & $85(7.8)$ & \\
\hline $100-$ & $0(0.0)$ & $1(0.1)$ & & $2(0.2)$ & $2(0.2)$ & \\
\hline Sex (female), $n(\%)$ & $615(50.1)$ & $891(47.4)$ & 0.05 & $545(50.1)$ & $542(49.8)$ & 0.01 \\
\hline ICU admission, $n$ (\%) & 449 (36.6) & $867(46.1)$ & 0.20 & $414(38.1)$ & $434(39.9)$ & 0.04 \\
\hline HCU admission, $n$ (\%) & $52(4.2)$ & $99(5.3)$ & 0.05 & $50(4.6)$ & $50(4.6)$ & $<0.001$ \\
\hline Hospital type (academic center), n (\%) & $240(19.6)$ & $606(32.3)$ & 0.29 & $239(22.0)$ & $206(18.9)$ & 0.08 \\
\hline Hospital volume, case/year mean (SD) & $5.5(4.9)$ & $7.2(7.4)$ & 0.27 & $5.7(5.1)$ & $5.7(5.2)$ & 0.01 \\
\hline \multicolumn{7}{|l|}{ Comorbidity, $n$ (\%) } \\
\hline Myocardial infarction & $16(1.3)$ & $17(0.9)$ & 0.04 & $11(1.0)$ & $9(0.8)$ & 0.02 \\
\hline Congestive heart failure & $70(5.7)$ & $130(6.9)$ & 0.05 & $65(6.0)$ & $58(5.3)$ & 0.03 \\
\hline Peripheral vascular disease & $16(1.3)$ & $26(1.4)$ & 0.01 & $14(1.3)$ & $13(1.2)$ & 0.01 \\
\hline Cerebrovascular disease & $58(4.7)$ & $72(3.8)$ & 0.04 & $47(4.3)$ & $46(4.2)$ & 0.01 \\
\hline Dementia & $34(2.8)$ & $48(2.6)$ & 0.01 & $28(2.6)$ & $27(2.5)$ & 0.01 \\
\hline Chronic pulmonary disease & $41(3.3)$ & $52(2.8)$ & 0.03 & $30(2.8)$ & $36(3.3)$ & 0.03 \\
\hline Mild liver disease & $37(3.0)$ & $70(3.7)$ & 0.04 & $33(3.0)$ & $32(2.9)$ & 0.01 \\
\hline Severe liver disease & $1(0.1)$ & $7(0.4)$ & 0.06 & $1(0.1)$ & $0(0.0)$ & 0.04 \\
\hline Rheumatologic disease & $30(2.4)$ & $42(2.2)$ & 0.01 & $25(2.3)$ & $24(2.2)$ & 0.006 \\
\hline Hemiplegia or paraplegia & $2(0.2)$ & $3(0.2)$ & 0.001 & $2(0.2)$ & $1(0.1)$ & 0.03 \\
\hline Diabetes without chronic complications & $120(9.8)$ & $182(9.7)$ & 0.003 & $104(9.6)$ & $95(8.7)$ & 0.03 \\
\hline Diabetes with chronic complications & $23(1.9)$ & $43(2.3)$ & 0.03 & $20(1.8)$ & $21(1.9)$ & 0.007 \\
\hline Renal disease & $71(5.8)$ & $183(9.7)$ & 0.15 & $69(6.3)$ & $69(6.3)$ & $<0.001$ \\
\hline Any malignancy, including leukemia and lymphoma & $207(16.9)$ & $300(16.0)$ & 0.02 & $188(17.3)$ & $184(16.9)$ & 0.01 \\
\hline Metastatic solid tumor & $66(5.4)$ & $96(5.1)$ & 0.01 & $61(5.6)$ & $58(5.3)$ & 0.01 \\
\hline \multicolumn{7}{|l|}{ Consciousness level, $n(\%)$} \\
\hline Alert & $925(75.4)$ & $1308(69.6)$ & 0.13 & $808(74.3)$ & $813(74.7)$ & 0.01 \\
\hline Delirium & $178(14.5)$ & $324(17.2)$ & 0.08 & $167(15.3)$ & $166(15.3)$ & 0.003 \\
\hline Somnolence & $50(4.1)$ & $100(5.3)$ & 0.06 & $43(4.0)$ & $48(4.4)$ & 0.02 \\
\hline Coma & $55(4.5)$ & $115(6.1)$ & 0.07 & $53(4.9)$ & $48(4.4)$ & 0.02 \\
\hline \multicolumn{7}{|l|}{ Intervention, $n(\%)$} \\
\hline Mechanical ventilation & $667(54.4)$ & $1258(67.0)$ & 0.26 & $625(57.4)$ & $638(58.6)$ & 0.02 \\
\hline Intermittent renal replacement therapy & $176(14.3)$ & $364(19.4)$ & 0.14 & $170(15.6)$ & $177(16.3)$ & 0.02 \\
\hline Continuous renal replacement therapy & $23(1.9)$ & $64(3.4)$ & 0.10 & $21(1.9)$ & $21(1.9)$ & $<0.001$ \\
\hline
\end{tabular}


Table 1 Baseline patient characteristics in unmatched and propensity-matched groups (Continued)

\begin{tabular}{|c|c|c|c|c|c|c|}
\hline \multirow[t]{3}{*}{ Variables } & \multicolumn{3}{|c|}{ Unmatched group } & \multicolumn{3}{|c|}{ Matched group } \\
\hline & \multirow{2}{*}{$\begin{array}{l}\text { H2RA group } \\
n=1227\end{array}$} & \multirow{2}{*}{$\begin{array}{l}\text { PPI group } \\
n=1879\end{array}$} & \multirow[t]{2}{*}{ SMD } & \multirow{2}{*}{$\begin{array}{l}\text { H2RA group } \\
n=1088\end{array}$} & \multirow{2}{*}{$\begin{array}{l}\text { PPI group } \\
n=1088\end{array}$} & \multirow[t]{2}{*}{ SMD } \\
\hline & & & & & & \\
\hline Polymyxin B hemoperfusion & $306(24.9)$ & $552(29.4)$ & 0.10 & $284(26.1)$ & $284(26.1)$ & $<0.001$ \\
\hline Central venous insertion & $350(28.5)$ & $783(41.7)$ & 0.28 & $335(30.8)$ & $345(31.7)$ & 0.02 \\
\hline \multicolumn{7}{|l|}{ Catecholamine, $n(\%)$} \\
\hline Dopamine & $948(77.3)$ & $1213(64.6)$ & 0.28 & $809(74.4)$ & $820(75.4)$ & 0.02 \\
\hline Noradrenaline & $660(53.8)$ & $1324(70.5)$ & 0.35 & $641(58.9)$ & $631(58.0)$ & 0.02 \\
\hline Vasopressin & $47(3.8)$ & $143(7.6)$ & 0.16 & $47(4.3)$ & $47(4.3)$ & $<0.001$ \\
\hline Adrenaline & $69(5.6)$ & $122(6.5)$ & 0.04 & $65(6.0)$ & $68(6.2)$ & 0.01 \\
\hline \multicolumn{7}{|l|}{ Transfusion, $n(\%)$} \\
\hline Red cell transfusion & 379 (30.9) & $733(39.0)$ & 0.17 & $361(33.2)$ & 369 (33.9) & 0.02 \\
\hline Platelets transfusion & $54(4.4)$ & $142(7.6)$ & 0.13 & $52(4.8)$ & $58(5.3)$ & 0.03 \\
\hline Fresh frozen plasma transfusion & $327(26.7)$ & $471(25.1)$ & 0.04 & $288(26.5)$ & $292(26.8)$ & 0.01 \\
\hline Antithrombin, $n(\%)$ & $245(20.0)$ & $463(24.6)$ & 0.11 & $234(21.5)$ & $232(21.3)$ & 0.004 \\
\hline Recombinant human soluble thrombomodulin, $n$ (\%) & $198(16.1)$ & $383(20.4)$ & 0.11 & $190(17.5)$ & $192(17.6)$ & 0.005 \\
\hline Immunoglobulin, $n(\%)$ & $457(37.2)$ & $735(39.1)$ & 0.04 & $428(39.3)$ & $393(36.1)$ & 0.07 \\
\hline Albumin, $n(\%)$ & $823(67.1)$ & $1369(72.9)$ & 0.13 & $738(67.8)$ & $753(69.2)$ & 0.03 \\
\hline Danaparoid, $n(\%)$ & $6(0.5)$ & $22(1.2)$ & 0.08 & $6(0.6)$ & $10(0.9)$ & 0.04 \\
\hline Low-molecular-weight heparin, $n(\%)$ & $11(0.9)$ & $27(1.4)$ & 0.05 & $11(1.0)$ & $14(1.3)$ & 0.03 \\
\hline Hydrocortisone, $n$ (\%) & 155 (12.6) & $340(18.1)$ & 0.15 & $145(13.3)$ & $145(13.3)$ & $<0.001$ \\
\hline \multicolumn{7}{|l|}{ Initial antibiotics use, $n(\%)$} \\
\hline Initial use of two or more & $342(27.9)$ & $568(30.2)$ & 0.05 & $320(29.4)$ & $329(30.2)$ & 0.02 \\
\hline Penicillin & $0(0.0)$ & $2(0.1)$ & 0.05 & $0(0.0)$ & $0(0.0)$ & $<0.001$ \\
\hline Ampicillin & $0(0.0)$ & $2(0.1)$ & 0.05 & $0(0.0)$ & $0(0.0)$ & $<0.001$ \\
\hline Ampicillin/sulbactam & $27(2.2)$ & $46(2.4)$ & 0.02 & $25(2.3)$ & $27(2.5)$ & 0.01 \\
\hline Piperacillin/tazobactam & $88(7.2)$ & $217(11.5)$ & 0.15 & $86(7.9)$ & $97(8.9)$ & 0.04 \\
\hline First-generation cephalosporin & $47(3.8)$ & $66(3.5)$ & 0.02 & $42(3.9)$ & $45(4.1)$ & 0.01 \\
\hline Second-generation cephalosporin & $499(40.7)$ & $573(30.5)$ & 0.21 & $418(38.4)$ & $419(38.5)$ & 0.002 \\
\hline Third-generation cephalosporin without effect for Pseudomonas aeruginosa & $43(3.5)$ & $53(2.8)$ & 0.04 & $40(3.7)$ & $41(3.8)$ & 0.01 \\
\hline Third-generation cephalosporin with effect for Pseudomonas aeruginosa & $3(0.2)$ & $8(0.4)$ & 0.03 & $3(0.3)$ & $3(0.3)$ & $<0.001$ \\
\hline Fourth-generation cephalosporin & $30(2.4)$ & $44(2.3)$ & 0.01 & $29(2.7)$ & $27(2.5)$ & 0.01 \\
\hline Carbapenem & $753(61.4)$ & $1306(69.5)$ & 0.17 & $703(64.6)$ & $690(63.4)$ & 0.03 \\
\hline Fluoroquinolone & $7(0.6)$ & $11(0.6)$ & 0.002 & $6(0.6)$ & $5(0.5)$ & 0.01 \\
\hline Aminoglycoside & $18(1.5)$ & $18(1.0)$ & 0.05 & $16(1.5)$ & $13(1.2)$ & 0.02 \\
\hline Clindamycin & $50(4.7)$ & $40(3.5)$ & 0.06 & $41(3.8)$ & $45(4.1)$ & 0.02 \\
\hline Tetracycline & $1(0.1)$ & $5(0.3)$ & 0.04 & $1(0.1)$ & $2(0.2)$ & 0.03 \\
\hline Macrolide & $0(0.0)$ & $7(0.4)$ & 0.09 & $0(0.0)$ & $0(0.0)$ & $<0.001$ \\
\hline Anti-MRSA drug & $20(1.6)$ & $62(3.3)$ & 0.11 & $20(1.8)$ & $20(1.8)$ & $<0.001$ \\
\hline Antifungal drug & $4(0.3)$ & $14(0.7)$ & 0.06 & $4(0.4)$ & $5(0.5)$ & 0.02 \\
\hline
\end{tabular}

H2RA histamine-2 receptor antagonists, PPI proton pump inhibiter, SD standard deviation, ICU intensive care unit, $H C U$ high care unit, MRSssA methicillin-resistant Staphylococcus aureus, SMD standardized mean difference

matching were assessed by standardized mean differences [22]. Absolute standardized mean differences of less than 0.1 were considered as negligible imbalances in the baseline characteristics between the groups
[23]. Fisher's exact test was used to compare outcomes between the two groups. We also estimated the treatment effect using inverse probability of treatment weighting (IPTW) using propensity scores. We 
calculated risk ratio, risk differences, and their 95\% confidence intervals (CI) between unmatched, propensity-matched, and IPTW analyses [24]. A $P$ value of less than 0.05 was considered statistically significant. Propensity score matching was performed using the "matching" package in the statistical software $\mathrm{R}$ version 3.1.3 (The $\mathrm{R}$ Foundation, Vienna, Austria). IPTW analyses were performed using the "survey" package in the statistical software $R$ version 3.1.3. All other analyses were performed using the IBM SPSS software version 25 (IBM SPSS, Armonk, NY).

\section{Results}

Overall, 3106 patients were identified during the study period. The H2RA group included 1227 patients, and the PPI group included 1879 patients. After one-to-one propensity score matching, 1088 pairs were created (Fig. 1).

Table 1 shows the baseline characteristics of the unmatched $(n=3106)$ and the propensity score-matched groups $(n=2176)$. After propensity score matching, the patients' backgrounds were well-balanced between the H2RA and PPI groups.

Table 2 shows the outcomes of the two groups. Before propensity score matching, there were no significant differences for gastrointestinal bleeding requiring endoscopic hemostasis, 28-day mortality, $\mathrm{CDI}$, and hospital-acquired pneumonia between the groups (Table 2).

After propensity score matching, there were no significant differences between the groups for gastrointestinal bleeding requiring endoscopic hemostasis within 28 days of admission $(0.74 \%$ vs $1.3 \%, P=0.284)$, 28 -day mortality (11.3\% vs $12.9 \%, P=0.386)$, CDI $(0.64 \%$ vs $0.46 \%, P=$ $0.774)$, and hospital-acquired pneumonia (3.0\% vs $4.3 \%$, $P=0.138$ ) (Table 2). Risk differences in the unmatched, propensity score-matched, and IPTW analysis groups showed similar results (Additional Table 2 and additional Table 3).

Table 3 shows risk ratios of the two groups. Before and after propensity score matching, there were no significant differences for gastrointestinal bleeding requiring endoscopic hemostasis, 28-day mortality, CDI, and hospital-acquired pneumonia between the groups (Table 3).

\section{Discussion}

In this retrospective study using a national inpatient database of Japan, there were no significant differences in gastrointestinal bleeding requiring endoscopic hemostasis within 28 days of admission, 28-day mortality, CDI, and hospital-acquired pneumonia between the H2RA and PPI groups in patients with septic shock after lower gastrointestinal perforation.

It is controversial whether H2RA increases gastrointestinal bleeding compared with PPI $[13,14]$. The effect of pharmacological acid suppression of H2RA was reported to be lower than that of PPI for treating active gastrointestinal bleeding [25]. However, H2RA reaches the target $\mathrm{pH}$ for stress ulcer prophylaxis within a day in approximately $65 \%$ of patients [26] and may be sufficient for stress ulcer prophylaxis. Thus, gastrointestinal bleeding requiring endoscopic hemostasis was not different between the groups.

It is unknown whether H2RA increases the risk of CDI compared with PPI. Gastric acid functions as a physiological barrier; however, H2RA and PPI altered these barrier mechanisms and were associated with bacterial overgrowth [27]. However, Clostridium species are usually acid-resistant and cells remain viable at gastric $\mathrm{pH}$ levels [28]; therefore, H2RA and/or PPI may not be associated with the increased risk of CDI. These points may also explain our results.

It is controversial whether H2RA increases the risk of hospital-acquired pneumonia compared with PPI. A gastric $\mathrm{pH}>4$ was associated with bacterial overgrowth and colonization and was associated with hospital-acquired pneumonia [29]. Although the pharmacological acid suppression of H2RA may be lower than that of PPI, both H2RA and PPI induced $\mathrm{pH}>4$ within 1 day of administration [26]. Therefore, the frequency of hospital-acquired pneumonia by H2RA may be similar to that of PPI.

Our study had several strengths. To the best of our knowledge, it is the first study to evaluate the effect

Table 2 Outcomes in the unmatched and propensity-matched groups

\begin{tabular}{|c|c|c|c|c|c|c|}
\hline & \multicolumn{3}{|c|}{ Unmatched groups } & \multicolumn{3}{|c|}{ Propensity-matched groups } \\
\hline & H2RA group & PPI group & $P$ & H2RA group & PPI group & $P$ \\
\hline Outcome, $n(\%)$ & $n=1227$ & $n=1879$ & & $n=1088$ & $n=1088$ & \\
\hline Gastrointestinal bleeding requiring endoscopic hemostasis & $8(0.65)$ & $25(1.33)$ & 0.076 & $8(0.74)$ & $14(1.3)$ & 0.284 \\
\hline 28-day mortality & $130(10.6)$ & $234(12.4)$ & 0.954 & $123(11.3)$ & $141(12.9)$ & 0.386 \\
\hline Clostridioides difficile infection & $10(0.81)$ & $12(0.64)$ & 0.663 & $7(0.64)$ & $5(0.46)$ & 0.774 \\
\hline Hospital-acquired pneumonia & $38(3.1)$ & $64(3.4)$ & 0.681 & $33(3.0)$ & $47(4.3)$ & 0.138 \\
\hline
\end{tabular}

H2RA histamine-2 receptor antagonists, PPI proton pump inhibiter 
Table 3 Risk ratios in the unmatched and propensity-matched groups

\begin{tabular}{lll}
\hline Outcomes & $\begin{array}{l}\text { Unmatched } \\
\text { groups }(95 \% \mathrm{Cl})\end{array}$ & $\begin{array}{l}\text { Propensity-matched } \\
\text { groups }(95 \% \mathrm{Cl})\end{array}$ \\
\hline $\begin{array}{l}\text { Gastrointestinal bleeding } \\
\text { requiring endoscopic } \\
\text { hemostasis }\end{array}$ & $0.49(0.22-1.08)$ & $0.57(0.24-1.36)$ \\
$\begin{array}{l}\text { 28-day mortality } \\
\text { Clostridioides difficile infection }\end{array}$ & $0.85(0.70-1.04)$ & $0.87(0.70-1.09)$ \\
Hospital-acquired pneumonia & $0.91(0.55-2.94)$ & $1.40(0.45-4.40)$ \\
\hline
\end{tabular}

Cl confidence interval

of H2RA compared with PPI in patients with septic shock after lower gastrointestinal tract perforation. Second, our study design was based on a real-world clinical setting and included approximately $50 \%$ of inpatients who were admitted to acute-care hospitals in Japan.

Our study had several limitations. First, the database lacks laboratory data such as lactate and clinical records including the results of cultures and susceptibility to peritonitis pathogens. Second, in Japan, patients with septic shock after lower gastrointestinal tract perforation were treated on general wards, and our results may not be generalized to other countries. Third, the present study could not assess mild-tomoderate gastrointestinal bleeding without requiring endoscopic hemostasis because the database did not include information regarding this status. Fourth, the database does not include information about initiation time of drug administration. Several procedures or drug administrations might have been performed before H2RA and PPI administration. Fifth, 28-day mortality in the present study was relatively low compared to those in previous studies. The difference may be due to exclusion of patients who died within 2 days of admission in the present study. Sixth, CDI or hospital-acquired pneumonia might have been underestimated, which could have led to less statistical power. Seventh, we defined sepsis using Angus criteria, which was validated in the DPC database. Although specificity of this definition was high, low sensitivity of sepsis may preclude extrapolation to other population. Last, although we used propensity score matching to adjust for patient backgrounds, unmeasured confounding factors might have biased our results.

\section{Conclusions}

In our study, H2RA were not associated with CDI, gastrointestinal bleeding requiring endoscopic hemostasis, 28day mortality, or hospital-acquired pneumonia compared with PPI in patients with septic shock after lower gastrointestinal tract perforation.

\section{Supplementary information}

Supplementary information accompanies this paper at https://doi.org/10. 1186/s40560-020-00473-0.

Additional file 1: Additional Table 1. ICD-10 codes to define sepsis. Additional file 2: Additional Table 2. Patient characteristsics in the IPTW analysis group.

Additional file 3: Additional Table 3. Risk differences for outcomes in the unmatched, propensity score-matched, and IPTW analysis groups.

\section{Abbreviations}

AIDS: Acquired immunodeficiency syndrome; CDI: Clostridioides difficile infection; Cl: Confidence interval; HCU: High care unit; HIV: Human immunodeficiency virus; H2RA: Histamine-2 receptor antagonists; ICD10: International Classification of Diseases, Tenth Revision; IPTW: Inverse probability of treatment weighting; MRSA: Methicillin-resistant Staphylococcus aureus; PPI: Proton pump inhibitor; SD: Standard deviation;

SMD: Standardized mean difference

\section{Acknowledgements}

Not applicable.

\section{Authors' contributions}

All authors contributed to the design of the study. JS and YS take responsibility for the integrity of the data and accuracy of the data analysis. JS wrote the manuscript and YS helped and revised the manuscript. SH, HM, TS, YM, TY, and HY directly participated in the planning, execution, or analysis of the study. All authors read and approved the final manuscript.

\section{Funding}

Funding Source: This work was supported by grants from the Ministry of Health, Labour and Welfare, Japan (H30-Policy-Designated-004 and H29-ICTGeneral-004); and Ministry of Education, Culture, Sports, Science and Technology, Japan (17H04141).

\section{Availability of data and materials}

Data cannot be made publicly available for ethical reasons because the data contains patient information. The study data are available to interested researchers upon reasonable request to the corresponding author, pending ethical approval.

\section{Ethics approval and consent to participate}

The study was approved by the Institutional Review Board of the University of Tokyo. Informed consent was waived because of the anonymous nature of the data.

\section{Consent for publication \\ Not applicable.}

\section{Competing interests}

The authors have disclosed that they do not have any potential conflicts of interest.

\section{Author details}

${ }^{1}$ Division of Infectious Diseases, Jichi Medical University Hospital, 3311-1 Yakushiji, Shimotsuke, Tochigi 329-0498, Japan. ${ }^{2}$ Data Science Center, Jichi Medical University, 3311-1 Yakushiji, Shimotsuke, Tochigi 329-0498, Japan. ${ }^{3}$ Division of General Medicine, Jichi Medical University, 3311-1 Yakushiji, Shimotsuke, Tochigi 329-0498, Japan. ${ }^{4}$ Department of Clinical Epidemiology and Health Economics, School of Public Health, The University of Tokyo, 7-3-1 Hongo, Bunkyo-ku, Tokyo 113-0033, Japan. ${ }^{5}$ Department of Infection and Immunity, School of Medicine, Jichi Medical University, 3311-1 Yakushiji, Shimotsuke, Tochigi 329-0498, Japan. 'Department of Clinical Laboratory Medicine, Jichi Medical University, 3311-1 Yakushiji, Shimotsuke, Tochigi 329-0498, Japan. 
Received: 26 February 2020 Accepted: 22 July 2020

Published online: 31 July 2020

\section{References}

1. Angus DC, Linde-Zwirble WT, Lidicker J, Clermont G, Carcillo J, Pinsky MR. Epidemiology of severe sepsis in the United States: analysis of incidence, outcome, and associated costs of care. Crit Care Med. 2001;29:1303-10.

2. Cruz DN, Antonelli M, Fumagalli R, Foltran F, Brienza N, Donati A, Malcangi V, Petrini F, Volta G, Bobbio Pallavicini FM, Rottoli F, Giunta F, Ronco C. Early use of polymyxin B hemoperfusion in abdominal septic shock: the EUPHAS randomized controlled trial. JAMA. 2009:301:2445-52.

3. Payen DM, Guilhot J, Launey Y, Lukaszewicz AC, Kaaki M, Veber B, Pottecher J, Joannes-Boyau O, Martin-Lefevre L, Jabaudon M, Mimoz O, Coudroy R, Ferrandière M, Kipnis E, Vela C, Chevallier S, Mallat J, Robert R, ABDOMIX Group. Early use of polymyxin B hemoperfusion in patients with septic shock due to peritonitis: a multicenter randomized control trial. Intensive Care Med. 2015;41:975-84

4. Solomkin JS, Mazuski JE, Bradley JS, Rodvold KA, Goldstein EJ, Baron EJ, O'Neill PJ, Chow AW, Dellinger EP, Eachempati SR, Gorbach S, Hilfiker M, May AK, Nathens AB, Sawyer RG, Bartlett JG. Diagnosis and management of complicated intra-abdominal infection in adults and children: guidelines by the Surgical Infection Society and the Infectious Diseases Society of America. Clin Infect Dis. 2010;50:133-64.

5. Lawson PA, Citron DM, Tyrrell KL, Finegold SM. Reclassification of Clostridium difficile as Clostridioides difficile (Hall and OToole 1935) Prévot 1938. Anaerobe. 2016:40:95-9.

6. Lessa FC, Mu Y, Bamberg WM, Beldavs ZG, Dumyati GK, Dunn JR, Farley MM, Holzbauer SM, Meek JI, Phipps EC, Wilson LE, Winston LG, Cohen JA, Limbago BM, Fridkin SK, Gerding DN, McDonald LC. Burden of Clostridium difficile infection in the United States. N Engl J Med. 2015;372:825-34.

7. Fernandez-Bustamante A, Frendl G, Sprung J, Kor DJ, Subramaniam B, Martinez Ruiz R, Lee JW, Henderson WG, Moss A, Mehdiratta N, Colwell MM, Bartels K, Kolodzie K, Giquel J, Vidal Melo MF. Postoperative pulmonary complications, early mortality, and hospital stay following noncardiothoracic surgery. JAMA Surg. 2017;152:157-66.

8. Mandell LA, Wunderink RG, Anzueto A, Bartlett JG, Campbell GD, Dean NC, Dowell SF, File TM Jr, Musher DM, Niederman MS, Torres A, Whitney CG, Infectious Diseases Society of America; American Thoracic Society. Infectious Diseases Society of America/American Thoracic Society consensus guidelines on the management of community-acquired pneumonia in adults. Clin Infect Dis. 2007:44:S27-72.

9. Oda S, Aibiki M, Ikeda T, Imaizumi H, Endo S, Ochiai R, Kotani J, Shime N, Nishida O, Noguchi T, Matsuda N, Hirasawa H, Sepsis Registry Committee of The Japanese Society of Intensive Care Medicine. The Japanese guidelines for the management of sepsis. J Intensive Care. 2014;2:55.

10. Rhodes A, Evans LE, Alhazzani W, Levy MM, Antonelli M, Ferrer R, Kumar A Sevransky JE, Sprung CL, Nunnally ME, Rochwerg B, Rubenfeld GD, Angus DC, Annane D, Beale RJ, Bellinghan GJ, Bernard GR, Chiche JD, Coopersmith C, De Backer DP, French CJ, Fujishima S, Gerlach H, Hidalgo JL, Hollenberg SM, Jones AE, Karnad DR, Kleinpell RM, Koh Y, Lisboa TC, Machado FR, Marini JJ, Marshall JC, Mazuski JE, McIntyre LA, McLean AS, Mehta S, Moreno RP, Myburgh J, Navalesi P, Nishida O, Osborn TM, Perner A, Plunkett CM, Ranieri M, Schorr CA, Seckel MA, Seymour CW, Shieh L, Shukri KA, Simpson SQ, Singer M, Thompson BT, Townsend SR, Van der Poll T, Vincent JL, Wiersinga WJ, Zimmerman $J$, Dellinger RP. Surviving sepsis campaign: international guidelines for management of sepsis and septic shock: 2016. Intensive Care Med. 2017:43:304-77.

11. Howell MD, Novack V, Grgurich P, Soulliard D, Novack L, Pencina M, Talmor D. latrogenic gastric acid suppression and the risk of nosocomial Clostridium difficile infection. Arch Intern Med. 2010;170:784-90.

12. Sasabuchi $Y$, Matsui H, Lefor AK, Fushimi K, Yasunaga H. Risks and benefits of stress ulcer prophylaxis for patients with severe sepsis. Crit Care Med. 2016:44:e464-9.

13. Alhazzani W, Alenezi F, Jaeschke RZ, Moayyedi P, Cook DJ. Proton pump inhibitors versus histamine 2 receptor antagonists for stress ulcer prophylaxis in critically ill patients: a systematic review and meta-analysis. Crit Care Med. 2013:41:693-705.

14. MacLaren R, Reynolds PM, Allen RR. Histamine-2 receptor antagonists vs proton pump inhibitors on gastrointestinal tract hemorrhage and infectious complications in the intensive care unit. JAMA Intern Med. 2014;174:564-74.
15. Matsuda S, Fujimori K, Kuwabara K, Ishikawa KB, Fushimi K. Diagnosis procedure combination as an infrastructure for the clinical study. Asian Pacific J Dis Manag. 2011;5:81-7.

16. Yamana H, Matsui H, Sasabuchi Y, Fushimi K, Yasunaga H. Categorized diagnoses and procedure records in an administrative database improved mortality prediction. J Clin Epidemiol. 2015;68:1028-35.

17. Matsuda S, Fujimori K, Fushimi K. Development of casemix based evaluation system in Japan. Asian Pacific J Dis Manag. 2010;4:55-66.

18. Yamana $H$, Horiguchi $H$, Fushimi $K$, Yasunaga $H$. Comparison of procedurebased and diagnosis-based identifications of severe sepsis and disseminated intravascular coagulation in administrative data. J Epidemiol. 2016;26:530-7.

19. Singer M, Deutschman CS, Seymour CW, Shankar-Hari M, Annane D, Bauer $M$, et al. The Third International Consensus Definitions for Sepsis and Septic Shock (Sepsis-3). JAMA. 2016;315:801-10.

20. Takagi K, Aoki M, Ishii T, Nagashima Y, Narita K, Nakagomi T, Tamura A, Yasui N, Hadeishi H, Taneda M, Sano K. Japan Coma Scale as a grading scale of subarachnoid hemorrhage: a way to determine the scale. No Shinkei Geka. 1998;26:509-15 [Article in Japanese].

21. Shigematsu $K$, Nakano $H$, Watanabe $Y$. The eye response test alone is sufficient to predict stroke outcome-reintroduction of Japan Coma Scale: a cohort study. BMJ Open. 2013;3.

22. Griswold ME, Russell Localio A, Mulrow C. Propensity score adjustment with multilevel data: setting your sites on decreasing selection bias. Ann Intern Med. 2010;152:393-6.

23. Austin PC. Balance diagnostics for comparing the distribution of baseline covariates between treatment groups in propensity-score matched samples. Stat Med. 2009;28:3083-107.

24. Austin PC. Variance estimation when using inverse probability of treatment weighting (IPTW) with survival analysis. Stat Med. 2016;35:5642-55.

25. Barkun AN, Bardou M, Pham CQ, Martel M. Proton pump inhibitors vs. histamine 2 receptor antagonists for stress-related mucosal bleeding prophylaxis in critically ill patients: a meta-analysis. Am J Gastroenterol. 2012;107:507-20.

26. Barkun AN, Cockeram AW, Plourde V, Fedorak RN. Acid suppression in nonvariceal acute upper gastrointestinal bleeding. Aliment Pharmacol Ther. 1999;13:1565-84.

27. Thorens J, Froehlich F, Schwizer W, Saraga E, Bille J, Gyr K, Duroux P, Nicolet M, Pignatelli B, Blum AL, Gonvers JJ, Fried M. Bacterial overgrowth during treatment with omeprazole compared with cimetidine: a prospective randomised double blind study. Gut. 1996;39:54-9.

28. Leffler DA, Lamont JT. Clostridium difficile Infection. N Engl J Med. 2015;372: 1539-48.

29. Apte NM, Karnad DR, Medhekar TP, Tilve GH, Morye S, Bhave GG. Gastric colonization and pneumonia in intubated critically ill patients receiving stress ulcer prophylaxis: a randomized, controlled trial. Crit Care Med. 1992; 20:530-9.

\section{Publisher's Note}

Springer Nature remains neutral with regard to jurisdictional claims in published maps and institutional affiliations.

Ready to submit your research? Choose BMC and benefit from:

- fast, convenient online submission

- thorough peer review by experienced researchers in your field

- rapid publication on acceptance

- support for research data, including large and complex data types

- gold Open Access which fosters wider collaboration and increased citations

- maximum visibility for your research: over $100 \mathrm{M}$ website views per year

At $\mathrm{BMC}$, research is always in progress.

Learn more biomedcentral.com/submission 